\title{
Comparison of CT and integrated PET-CT based radiation therapy planning in patients with malignant pleural mesothelioma Berrin Pehlivan*1, Erkan Topkan ${ }^{\dagger 1}$, Cem Onal ${ }^{\dagger 1}$, Gul Nihal Nursal ${ }^{\dagger 2}$, Oznur Yuksel ${ }^{\dagger 1}$, Yemliha Dolek ${ }^{\dagger 1}$, Melek Nur Yavuz ${ }^{\dagger 1}$ and Ali Aydin Yavuz ${ }^{\dagger 1}$
}

\author{
Address: ${ }^{1}$ Department of Radiation Oncology, Baskent University Medical Faculty, Adana Medical and Research Center, Kisla Campus, Adana, \\ Turkey and 2Department of Nuclear Medicine, Baskent University Medical Faculty, Adana Medical and Research Center, Kisla Campus, Adana, \\ Turkey \\ Email: Berrin Pehlivan* - berrin_pehlivan@yahoo.com; Erkan Topkan - drerkantopkan@yahoo.com; Cem Onal - hcemonal@hotmail.com; \\ Gul Nihal Nursal - gnnursal@yahoo.com; Oznur Yuksel - droznuryuksel@hotmail.com; Yemliha Dolek - yemliha2@hotmail.com; \\ Melek Nur Yavuz - myavuz@baskent-adn.edu.tr; Ali Aydin Yavuz - ayavuz@ baskent-adn.edu.tr \\ * Corresponding author †Equal contributors
}

Published: 16 September 2009

Radiation Oncology 2009, 4:35 doi:10.1/86/1748-7/7X-4-35
Received: I July 2009

Accepted: 16 September 2009

This article is available from: http://www.ro-journal.com/content/4/I/35

(c) 2009 Pehlivan et al; licensee BioMed Central Ltd.

This is an Open Access article distributed under the terms of the Creative Commons Attribution License (http://creativecommons.org/licenses/by/2.0), which permits unrestricted use, distribution, and reproduction in any medium, provided the original work is properly cited.

\begin{abstract}
Background: When combined with adequate tumoricidal doses, accurate target volume delineation remains to be the one of the most important predictive factors for radiotherapy (RT) success in locally advanced or medically inoperable malignant pleural mesothelioma (MPM) patients. Recently, 18-fluorodeoxyglucose positron emission tomography (PET) has demonstrated significant improvements in diagnosis and accurate staging of MPM. However, role of additional PET data has not been studied in RT planning (RTP) of patients with inoperable MPM or in those who refuse surgery. Therefore, we planned to compare CT with co-registered PET-CT as the basis for delineating target volumes in these patients group.
\end{abstract}

Methods: Retrospectively, the CT and co-registered PET-CT data of 13 patients with histologically proven MPM were utilized to delineate target volumes separately. For each patient, target volumes (gross tumor volume [GTV], clinical target volume [CTV], and planning target volume [PTV]) were defined using the CT and PET-CT fusion data sets. The PTV was measured in two ways: PTVI was CTV plus a I-cm margin, and PTV2 was GTV plus a I-cm margin. We analyzed differences in target volumes.

Results: In 12 of 13 patients, compared to CT-based delineation, PET-CT-based delineation resulted in a statistically significant decrease in the mean GTV, CTV, PTVI, and PTV2. In these 12 patients, mean GTV decreased by $47.1 \% \pm 28.4 \%$, mean CTV decreased by $38.7 \% \pm 24.7 \%$, mean PTVI decreased by $31.1 \% \pm 23.1 \%$, and mean PTV2 decreased by $40.0 \% \pm 24.0 \%$. In 4 of 13 patients, hilar lymph nodes were identified by PET-CT that was not identified by CT alone, changing the nodal status of tumor staging in those patients.

Conclusion: This study demonstrated the usefulness of PET-CT-based target volume delineation in patients with MPM. Co-registration of PET and CT information reduces the likelihood of geographic misses, and additionally, significant reductions observed in target volumes may potentially allow escalation of RT dose beyond conventional limits potential clinical benefits in tumor control rates, which needs to be tested in future studies. 


\section{Background}

Malignant pleural mesothelioma (MPM) is a relatively rare but highly aggressive tumor with expected median survival of only 9 to 17 months $[1,2]$. Although currently it appears to be a rare tumor, its incidence is increasing throughout most of the world including Turkey, where it is epidemic in three villages of the Cappadocia region. Also, familial forms with autosomal dominant inheritance have been reported in this region $[3,4]$.

Although there is no universally accepted standard treatment for MPM, currently the EPP is the most widely preferred treatment modality. However, due to significant procedure related modality and mortality 85 to $90 \%$ of patients are not eligible for this aggressive procedure $[5,6]$. In this context, radiation therapy (RT) as the sole treatment in the presence or absence of concurrent chemotherapy may be a good alternative in suitable patient population. However, RT planning (RTP) for MPM is difficult due to the large, irregularly shaped area at risk, the high doses required for local control, and the proximity of many radiosensitive structures such as the liver, ipsilateral kidney, heart, spinal cord, esophagus, contralateral lung, and the ipsilateral lung itself in inoperable cases. In the latter setting, which is a therapeutic challenge, the recent, more sophisticated RT techniques, including intensitymodulated radiotherapy (IMRT), image guided radiotherapy (IGRT), and especially helical tomotherapy (HT), are promising. However, similar with all other tumor sites, accurate target delineation is crucial when RT is considered as the sole treatment or as a component of oncologic treatment, and additionally when combined with adequate tumoricidal doses, accurate target volume delineation remains to be the one of the most important predictive factors for RT success in MPM.

Computed tomography (CT) is the primary imaging modality used in staging and RT planning for MPM. Rindlike extension of the tumor on the pleural surfaces is the most common CT feature [7]. However, CT often fails to accurately demonstrate transdiaphragmatic invasion and mediastinal lymph nodes [8,9]. Recently, 18-fluorodeoxyglucose positron emission tomography (PET) has demonstrated significant improvements in diagnosis, accurate staging, RTP, and assessment of tumor response to the prescribed treatment in a variety of tumor sites including the MPM [10-16]. PET imaging is based on biochemical processes that may offer better detection of tumors even before they become anatomically apparent. Integration of functional PET data with the detailed anatomical information of CT (PET-CT) has markedly increased the sensitivity, specificity and accuracy of discrimination between benign and malignant diseases, determination of tumoral extensions in to the mediastinum, abdominal cavity or pleural surfaces, medistinal lymph nodes or distant vis- cera $[10,17,18]$. In this context, integrated PET-CT provides more information, compared with ametabolic CT or nonanatomic PET. The high sensitivity and specificity of PET-CT in patients with MPM have been well documented. Benard et al analyzed 28 patients with suspected MPM and reported that specificity of PET was $100 \%$ with sensitivity of $91 \%$ in differentiating benign and malignant lesions [10]. Caretta et al found similar results, with accuracy of PET at $92 \%$ in the differential diagnosis of pleural diseases [17]. Similarly, in their recent report Plathow et al analyzed 54 patients with stage II and III MPM, and the authors reported accuracy of $77 \%$ for CT, $86 \%$ for PET, $80 \%$ for Magnetic Resonance Imaging (MRI), and 100\% for PET-CT in patients with stage II disease, and accuracy of $75 \%$ for CT, $83 \%$ for PET, $90 \%$ for MRI, and $100 \%$ for PET-CT in patients with stage III disease [18].

To our knowledge, no studies address the role of PET-CTbased RTP in patients with medically inoperable MPM or in those who refuse surgery. Largely based on the aforementioned data, we hypothesized that using PET-CT data rather than CT data alone would change RT fields and possibly result in fewer geographic misses for unresected MPM. Therefore, in the present study, we compared CTbased and integrated PET-CT-based gross tumor volume (GTV) delineation and its subsequent expansion to clinical target volume (CTV) and planning target volume (PTV).

\section{Methods}

Thirteen patients with histological diagnosis of MPM who were not candidate for a curative resection due to medical reasons or self refusal those who were treated with thorasic irradiation with a palliative intent are planned to be reassessed whether the intented target volumes may have changed if additional PET data was used in conjunction with CT compared to CT alone. This study was largely based on recent impressive high sensitivity and specifity data of PET in MPM diagnosis and staging as mentioned previously $[10,17,18]$. This pure delineation study protocol to evaluate the potential differences via implementation of PET-CT on palliative MPM cases was approved by the institutional ethic committee. Patients' charts were reviewed for the search of characteristics with nonmetastatic mesothelioma classified as T2-4 and/or N0-3 according to the International Mesothelioma Interest Group staging system [19], and no previous surgical resection.

As we acknowledged from patients' hospital records, each patient was placed in the supine position with both arms raised above their heads in a manner identical to treatment positioning during PET-CT. The PET-CT scan was performed in an integrated PET-CT system (Discovery-STE 8, General Electric System, Milwaukee, WI, USA). Patients 
were advised to fast for at least 6 hours before the PET appointment. After 370 to $555 \mathrm{MBq}(10-15 \mathrm{mCi})$ 18fluorodeoxyglucose was injected, patients rested for approximately 60 minutes in a comfortable chair. Preinjection blood glucose levels were measured to ensure that they were below $150 \mathrm{mmol} / \mathrm{L}$. The patients were scanned on a flat-panel carbon fiber composite table insert. An enhanced CT scan from the base of the skull to the inferior border of the pelvis was acquired with 5-mm slice thickness, using a standardized protocol with $140 \mathrm{kV}$ and 80 MA with contrast injection. The subsequent PET scan was acquired from the base of the skull to the inferior border of the pelvis as in the CT scan, using multiple-bed position. Attenuation was corrected by using the CT images. The processed images were displayed in coronal, transverse, and saggital planes.

After image acquisition, PET-CT data sets were transferred to our treatment planning system, Eclipse 7.5 (Varian Medical Systems, Palo Alto, CA, USA) into DICOM RT format, and the available data was utilized for planning purposes following image fusion. The CT-based and PET-CTbased treatment planning was computed for each patient. The target volumes were defined by the radiation oncologist (BP and checked by ET) with specific experience in MPM cancer treatment on the CT and integrated PET-CT images. The GTV was defined as the volume of macroscopic primary tumor and involved hilar and mediastinal lymph nodes identified on the planning CT. The CTV was created automatically with a $1-\mathrm{cm}$ margin around the GTV with respecting to the natural anatomical barriers, such as vertebral column. The PTV1 encompassed the CTV plus a mean $1-\mathrm{cm}$ margin, and PTV2 was created with a $1-\mathrm{cm}$ margin to the GTV. All volumes were defined again on integrated PET-CT images. Lungs (right and left separately), liver, heart, esophagus, and kidneys (right and left) were counted as organs at risk in each patient. We set the window and level for the PET images according to method previously described by Erdi et al for accurate target volume definition [20]. In this protocol, we first measured the value of the hottest pixel in the lesion and then set the upper window level to this maximum value and set the lower window level to $42 \%$ of the maximum level.

\section{Statistical Analysis}

On the basis of the literature, we hypothesized that integration of PET into RTP would change the target volumes in approximately $30 \%$ of the patients. To detect such a change with a $95 \%$ confidence interval of $5 \%$ to $55 \%$, we needed to enroll at least 13 patients. Statistical differences between paired parameters from CT-based versus PET-CTbased treatment plans were evaluated with the Wilcoxon signed rank test. Results are expressed as mean \pm standard deviation (SD). Differences were considered statistically significant when the two-tailed $P$ value was less than .05 .

\section{Results}

Demographic and clinical characteristics of the 13 patients are depicted in Table 1. Four of the 13 patients were women. Median age was 50 years, with range of 38 to 74 years. In all but one patient, compared with CTbased delineation, PET-CT-based delineation resulted in significantly decreased mean GTV, CTV, PTV1, and PTV2 (Table 2). In these 12 patients, mean GTV decreased by $47.1 \% \pm 28.4 \%$, mean CTV decreased by $38.7 \% \pm 24.7 \%$, mean PTV1 decreased by $31.1 \% \pm 23.1 \%$, and mean PTV2 decreased by $40.0 \% \pm 24.0 \%$. In all 12 patients the respective target volume reductions were solely due to reduced primary tumor volumes on PET-CT fusion compared to CT with no change in nodal disease exclusion by PET data. In one patient, volumes were increased by PET-CT compared with CT; these increases were $19 \%, 2 \%, 10 \%$ and 15\% in GTV, CTV, PTV1 and PTV2, respectively. This

Table I: Patient characteristics.

\begin{tabular}{|c|c|c|c|c|c|c|}
\hline Patient \# & Sex & Age, y & ECOG & Stage & $\mathbf{S U V}_{\text {max }}$ & SUVmean \\
\hline I & Male & 50 & I & T4N3M0 & 11.5 & 7.5 \\
\hline 2 & Female & 54 & I & T3NIMO & 21.8 & 12.5 \\
\hline 3 & Male & 74 & 0 & TIaN3M0 & 10.9 & 5.1 \\
\hline 4 & Male & 38 & I & T4NIM0 & 4.2 & 2.3 \\
\hline 5 & Male & 43 & 0 & TlaN2 M0 & 6.7 & 3.5 \\
\hline 6 & Male & 47 & 0 & T2NOMO & 6.5 & 4.1 \\
\hline 7 & Male & 42 & 0 & TIaN3M0 & 7.2 & 6.9 \\
\hline 8 & Female & 71 & I & TIaN3M0 & 9.8 & 5.6 \\
\hline 9 & Male & 46 & 0 & T3NOMO & 13.2 & 8.6 \\
\hline 10 & Female & 64 & I & TIaN2M0 & 7.9 & 4.7 \\
\hline I I & Male & 74 & 0 & TIaN2M0 & 15.3 & 8.4 \\
\hline 12 & Male & 47 & I & $\mathrm{T} 4 \mathrm{~N} 2 \mathrm{M} 0$ & 12.0 & 6.9 \\
\hline 13 & Female & 50 & 0 & T3NIMO & 9.5 & 6.8 \\
\hline
\end{tabular}

*Abbreviations: ECOG, Eastern Cooperative Oncology Group; M, distant metastasis; N, lymph-nodal disease; T, tumor extension. Stage was determined using the International Mesothelioma Interest Group criteria. 
Table 2: Volumes by CT and PET-CT in I 3 Patients with Malignant Pleural Mesothelioma.

\begin{tabular}{llll}
\hline Volume, cc & CT & PET-CT & P Value \\
\hline GTV & & & \\
Mean \pm S.D. & $788.9 \pm 845.1$ & $441.4 \pm 420.0$ & 0.01 \\
$\begin{array}{l}\text { Min-max } \\
\text { CTV }\end{array}$ & $(101.4-3352.1)$ & $(38.5-1250.2)$ & \\
Mean \pm S.D. & $2040.6 \pm 1360.5$ & $1533.1 \pm 1483.6$ & 0.002 \\
Min-max & $(479.6-5615.8)$ & $(254.4-5615.82)$ & \\
PTV I & & & \\
Mean \pm S.D. & $3824.7 \pm 1777.7$ & $2936.7 \pm 1940.1$ & 0.003 \\
Min-max & $(1062.5-7523.8)$ & $(608.9-6971.9)$ & \\
PTV2 & & & \\
Mean \pm S.D. & $2385.5 \pm 1449.9$ & $1627.9 \pm 1254.2$ & 0.003 \\
Min-max & $(488.2-5853.1)$ & $(278.9-4088.4)$ & \\
\hline
\end{tabular}

*Abbreviations: $\mathrm{CT}=$ computed tomography; CTV = clinical target volume; GTV = gross tumor volume; $\mathrm{PET}-\mathrm{CT}=$ positron emission tomography-computed tomography; PTVI = planning target volume I (defined as CTV plus a I-cm margin); PTV2 = planning target volume 2 (defined as GTV plus a I-cm margin).

increament was due to additional involved lymph node detection by PET data which was not appearent on CT.

In 4 of 13 patients (31\%), PET-CT identified increased 18 fluorodeoxyglucose uptake in hilar lymph nodes that did not appear on $\mathrm{CT}$, thereby changing the $\mathrm{N}$ stage in those 4 patients. In 3 patients $(23 \%)$, PET-CT showed subdiaphragmatic extension of the disease which did not appear on CT. Representative images of a patient with different GTV delineations are seen in the Figure 1 and Figure 2.

\section{Discussion}

On background of a nonexistent radiotherapeutic consensus for unresected nonmetastatic MPM in the literature, we performed a pure delineation study to evaluate the differences via implementation of PET-CT in order to generate potential possibilities for future radiotherapy decisions with current and coming cutting edge technologic advances. The results of the current study revealed that compared to CT, integrated PET-CT-based target volume delineation significantly reduced the GTV and its expansions, CTV and PTV, in 12 of 13 patients and increased target volumes in 1 patient, all together impacting the importance of accurate target volume delineation in this patients group. Additionally, we found that functional PET data changed the $\mathrm{N}$ stage in 4 of 13 patients, and subdiaphragmatic tumor extension was evident in further $3(23 \%)$ patients that was not shown by CT, which may explain the possibility of geographic misses experienced with CT-based RTP and its influence on poor outcomes in patients with MPM.

There is currently no universally accepted standard therapy for MPM. Regarding the difficulties in diagnosis, stag- ing, and treatment, it presents a unique therapeutic challenge. Currently, EPP with en bloc resection of the lung, pleura, ipsilateral diaphragm, and pericardium is the treatment of choice. However, only $10 \%$ to $15 \%$ of patients are eligible for this extensive surgery $[5,6]$, and significant procedure-related morbidity and mortality limit its use. In addition, even with EPP, R0 resection is theoretically impossible, and microscopic or macroscopic disease almost always remains at the resection margins. When EPP is the sole treatment modality, locoregional recurrence is unacceptably high, ranging from $31 \%$ to $64 \%$ [21-23]. Therefore, postoperative RT is usually indicated. In a number of studies, high-dose hemithoracic RT of 45 to 50 Gy with a boost to 54 to 60 Gy targeted to areas at higher risk for local recurrence significantly improved local control [24-27]. In a study by Perrot et al, only $10 \%$ of patients developed recurrence in the ipsilateral hemithorax after completion of intended 60 Gy RT [24]. Similarly, Rusch et al demonstrated that adjuvant hemithoracic RT of $54 \mathrm{~Gy}$ following EPP improved local control with a $13 \%$ risk of local failure [26]. However, as was the case in our current cohort, the majority of patients with malignant pleural mesothelioma are not good candidates for curative EPP due to presence of either advanced local disease or unfavorable medical conditions that render them unfit for surgery.

In the setting of unresectable or medically inoperable/ patient refusal conditions, RT when applied with pallative intent may offer good symptom control in conventional palliative doses. However, there is strong evidence suggesting better symptom and possibly loco-regional tumor control with higher doses approaching to that is used for curative intent. In one study, Ball et al showed that only 1 $(4 \%)$ of 23 patients who received $<40$ Gy achieved symptomatic relief while $4(66 \%)$ of 6 patients treated with $>$ 40 Gy had satisfactory symptom palliation impacting the importance of total dose even for palliative purposes [28]. Largely based on this data we planned to reassess our patients those who were treated with an palliative approach whether they were suitable for higher RT doses in the range of curative $54 \mathrm{~Gy}$, as these patients theoratically still bear a chance for cure with higher RT doses even in absence of EPP. However, absence of a HT unit or a similar volumetric arc technology in our clinics significantly limited our ability to create clinically relevant and acceptable RTPs based on compatible pulmonary toxicity criteria. Therefore we planned to only compare the conventional CT- and PET-CT based target volume delineations which may positively impact and alter the future RTPs either for curatively or palliatively intended approaches in presence of HT facilities.

Despite the evident advantages offered by escalated doses with use of 3D-conformal RT it is not usually possible to 

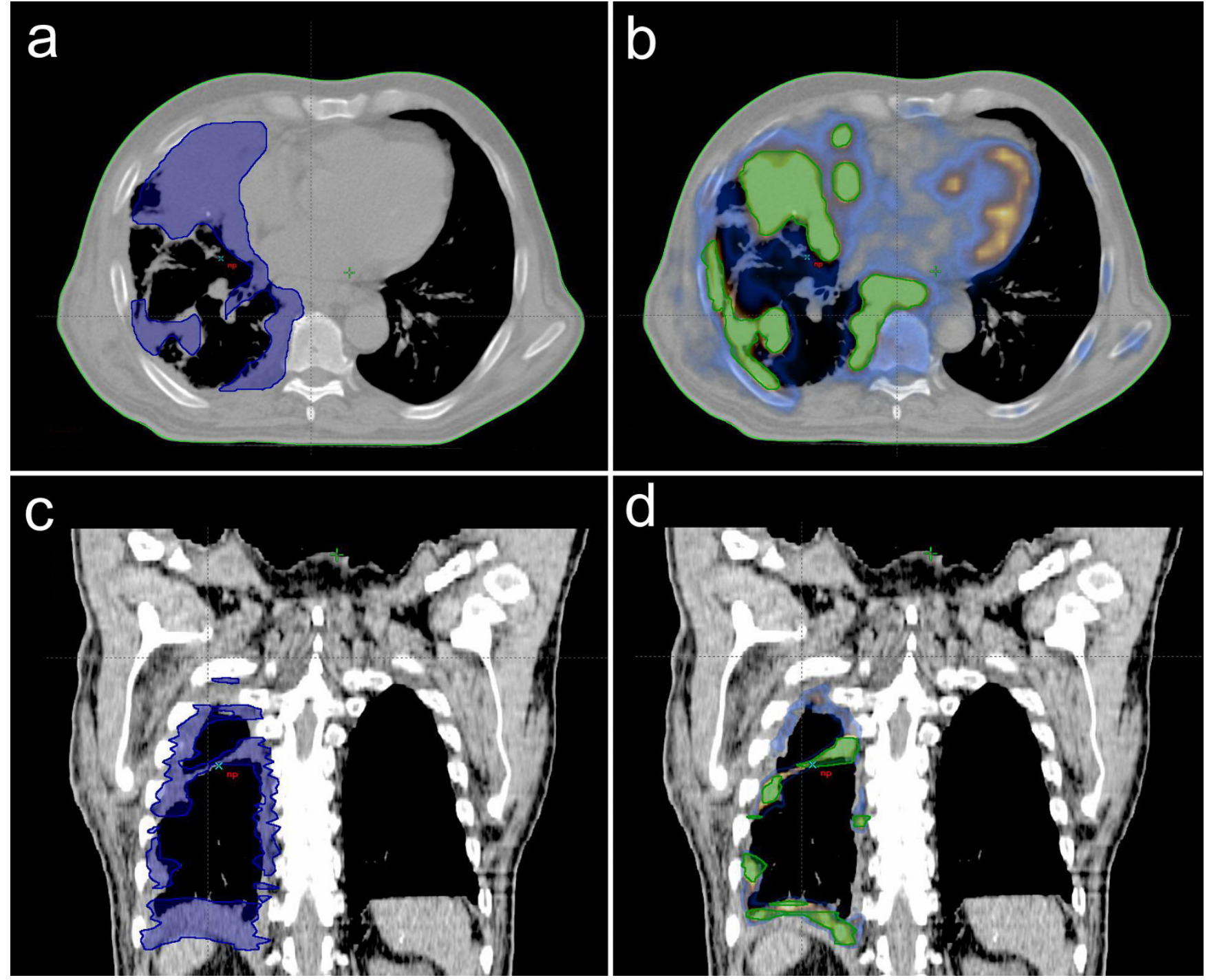

Figure I

Representative image of a patient with CT- and PET- CT based GTV delineations; (a) axial CT (b) axial PETCT, (c) coronal CT, (d) coronal PET-CT. *Abbreviations: GTV = gross tumor volume; CT = computed tomography; PET$\mathrm{CT}=$ positron emission tomography-computed tomography.

escalate RT dose because of significant toxicity concerns Linden et al treated 47 MPM patients with a dose of $40 \mathrm{~Gy}$ in 20 fractions with and without chemotherapy and they informed that all of patients experienced radiation induced pulmonary fibrosis [29]. However, in this setting, more sophisticated RT techniques such as IMRT, IGRT, and especially helical-slit IMRT (HT) and cone-beam IMRT (RapidArc and VMAT) [30] might become appropriate alternatives for either definitive or palliative treatment for suitable patients based on compatible pulmonary toxicity criteria. Helical tomotherapy is a promising method, and achieves a better dose conformity in several tumor sites including MPM [31-33]. In their recent study, Ster- zing et al compared step-and-shoot IMRT with HT [33]. They observed that while both modalities achieved excellent dose distributions, target coverage and homogenity could be increased significantly with HT, additionally contralateral lung dose could be lowered beyond 5 Gy. They concluded that HT is an excellent option for the IMRT of MPM. In our current study, as aforementioned it was impossible to create appropriate RTPs and guiding DVHs in absence of further technical advances such as HT oppurtunities, yet, we believe that the significant reductions observed in target volumes, additional subdiaphragmatic extension and involved lymph nodes shown only 
by PET data might be accepted as a useful evidence for future studies with appropriate technologies.

Although CT is the primary imaging modality for both staging and RTP in patients with MPM, the results of CT fail to identify the true extent of local invasion through the extrathoracic fascia, diaphragmatic surfaces, and interlobar fissures. Results of CT cannot accurately distinguish between malignant and benign conditions, such as inflammation and pleural fluid, which are common findings of MPM. Webb et al showed that the desmoplastic reaction caused by tumor-induced proliferation of benign connective tissue adjacent to the tumor can result in an overestimation of the stage of the tumor [34]. Additionally, CT has poor sensitivity for defining the malignant status of mediastinal lymph nodes [8,9]. Earlier studies in lung cancer showed significant changes when PET information was applied, with decreased volumes mostly attributed to exclusion of atelectasis [15,35-37]. We found that 18-fluorodeoxyglucose PET led to better definition of target volumes with additional metabolic information, and it was more successful in discriminating between tumor and benign connective tissue changes.

The additional volume and intratumoral functional variations uniquely identified by PET may be even more important in the near future when so-called dose-painting intensity-modulated radiotherapy becomes widely used in clinical practice, opening the possibility of controlled and reproducible internal-dose escalation to functionally important areas of the tumor. With the use of more specific functional PET tracers, this high-precision RT technique could help enormously in resolving the problems of overestimation and underestimation of GTV and mitigate their negative consequences for radiation management of tumors at many sites, including MPM.

We believe that our current study might be a significant contribution to the emerging RT literature regarding the use of PET-CT data in conjunction with CT in RTP of MPM patients. However, it is not appropriate to draw strict conclusions based on the current results without conformation of its use with novel sophisticated RT techniques such as HT with dose- volume histogram data which can predict RT related toxicity after curative or palliative RT. Therefore the present study seems to be a baseline data for further clinical and dosimetric studies rather than being considered as a guide.

\section{Conclusion}

This study demonstrated the usefulness of PET/CT based target volume delineation in patients with MPM. The largest potential benefit of incorporating PET into RTP for MPM may be the reduction in geographic misses associated with CT-based planning, and, as a result, the poten- tial reduction in local and regional treatment failures. However, we believe that before reaching more definite conclusions, more clinical studies are required to better define the role of PET-CT fusion in this setting.

\section{Competing interests}

We have no personal or financial conflict of interest and have not entered into any agreement that could interfere with our access to the data on the research, or upon our ability to analyze the data independently, to prepare manuscripts, and to publish them.

\section{Authors' contributions}

All authors read and approved the final manuscript. BP and ET carried out all CT evaluations, study design, target delineations, interpretation of the study, and drafted the manuscript. GNN carried out all PET evaluations and delineation of target volumes based on PET findings. CO carried out statistical analysis. OZ participated in manuscript preparation and study design. YD made the treatment planning. MNY, AAY gave advice on the work and helped in the interpretation of the data.

\section{Acknowledgements}

We would thank to Dr Ali Fuat Yapar (AFY) for revision of PET data.

\section{References}

I. Ruffie P, Feld R, Minkin S, Cormier Y, Boutan-Laroze A, Ginsberg R, Ayoub J, Shepherd FA, Evans WK, Figueredo A: Diffuse malignant mesothelioma of the pleura in Ontario and Quebec: $A$ retrospective study of 332 patients. J Clin Oncol 1989, 7: I I57-I I68.

2. Martino D, Pass HI: Integration of multimodality approaches in the management of malignant pleural mesothelioma. Cli Lung Cancer 2004, 5:290-298.

3. Dogan AU, Barýs YI, Dogan M, Emri S, Steele I, Elmishad AG, Carbone $M$ : Genetic predisposition to fiber carcinogenesis caues a mesothelioma epidemic in Turkey. Cancer Res 2006, 66:5063-5068.

4. Roushdy-Hammady I, Siegel J, Emri S, Testa JR, Carbone M: Geneticsuspectibility factor and malignant mesothelioma in the Cappodocian region of Turkey. Lancet 200 I, 357:444-445.

5. Yoshino I, Yamaguchi M, Okamoto T, Ushijima C, Fukuyama Y, Ichinose $Y$, Maehara $Y$ : Multimodel treatment for resectable epithelial type malignant pleural mesothelioma. World J Surg Oncol 2004, 2: II.

6. Sugarbaker D, Flores R, Jaklitsh M, Richards WG, Strauss GM, Corson JM, DeCamp MM Jr, Swanson SJ, Bueno R, Lukanich JM, Baldini EH, Mentzer SJ: Resection margins, extrapleural nodal status and cell type determine postoperative long-term survival in trimodality therapy of malignant pleural mesothelioma: results in I 83 patients. J Thorac Cardiovasc Surg 1999, I:54-63.

7. Metintas M, Ucgun I, Elbek O, Erginel S, Metintas S, Kolsuz M, Harmancý E, Alatas F, Hillerdal G, Ozkan R, Kaya T: Computed tomography features in malignant pleural mesothelioma and other commonly seen pleural diseases. Eur J Radiol 2002, 4l: I-9.

8. Rusch VW, Godwin JD, Shuman WP: The role of computed tomography scanning in the initial assesment and the follow-up of malignant pleural mesothelioma. J Thorac Cardiovasc Surg 1988, 96: I7I-7.

9. Heelan RT, Rusch VW, Begg CB, Panicek DM, Caravelli JF, Eisen C: Staging of malignant pleural mesothelioma: comparison of CT and MR imaging. AJR Am J Roentgenol 2004, 4: I 039- I047.

10. Bénard F, Sterman D, Smith RJ, Kaiser LR, Albelda SM, Alavi A: Metabolic imaging of malignant mesothelioma with fluorodeox- 
yglucose positron emission tomography. Chest 1998, I 1 4:7|3-22.

II. Kluetz PG, Meltzer CC, Villemange VL, Kinahan PE, Chander S, Martinelli MA, Townsend DW: Combined PET/CT imaging in oncology: Impact on patient management. Clin Positron Imaging 2000, 3:223-230.

12. Bar-Bar-Shalom R, Yefremov N, Guralnik L, Gaitini D, Frenkel A, Kuten A, Altman H, Israel O: Clinical performance of PET/CT in evaluation of cancer: additional value for diagnostic imaging and patient management. J Nucl Med 2003, 44:1200-1 209.

13. Ciernik F, Dizendorf E, Baumert BG, Reiner B, Burger C, Davis JB, Lütolf UM, Steinert HC, Von Schulthess GK: Radiation treatment planning with an integrated positron emission and computer tomography (PET/CT): A feasibility study. Int J Radiat Oncol Biol Phys 2003, 57:853-863.

14. Roman CD, Martin WH, Delbeke D: Incremental value of fusion imaging with integrated PET-CT in oncology. Clin Nucl Med 2005, 30:470-477.

15. van Baardwick A, Baumert BG, Bosmans G, van Kroonenburgh M, Stroobants S, Gregoire V, Lambin P, De Ruysscher D: The current status of PET-FDG in tumor voulme definition in radiotherapy treatment planning. Cancer Treat Rev 2006, 32:245-60.

16. Topkan E, Yavuz AA, Aydin M, Onal C, Yapar F, Yavuz MN: Comparison of CT and PET-CT based planning of radiation therapy in locally advanced pancreatic carcinoma. J Cancer Exp Clin Cancer Res 2008, 27:4I.

17. Caretta A, Landoni C, Melloni G, Ceresolli GL, Compierchio A, Fazio F, Zannini P: I 8-FDG PET in the evaluation of malignant pleural disease: a pilot study. Eur J Cardiovasc Surg 2000, 17:377-383.

18. Plathow C, Staab A, Schmaehl A, Schmaehl A, Aschoff P, Zuna I, Pfannenberg C, Peter SH, Eschmann S, Kloop M: Computed tomography, positron emission tomograpy, positron emission tomograpy/computed tomography, and magnetic resonance imaging for staging of limited pleural meothelioma. Invest Radiol 2008, 43:737-744

19. International Mesothelioma Interest Group: A proposed new international TNM staging system for malignant pleural mesothelioma. Chest 1995, 108: I 122-8.

20. Erdi YE, Rosenzweig K, Erdi AK, Macapinlac HA, Hu YC, Braban LE, Humm JL, Squire OD, Chui CS, Larson SM, Yorke ED: Radiotherapy treatment planning for patients with non-small cell lung cancer using positron emission tomography (PET). Radiother Oncol 2002, 62:5I-60.

21. Sugarbaker DJ, Garcia JP, Richards WG, Harpole DH Jr, Healy-Baldini E, DeCamp MM Jr, Mentzer SJ, Liptay MJ, Strauss GM, Swansob S]: Extrapleural pneumonectomy in the multimodality therapy of malignant pleural mesothelioma: Results in 120 consecutive patients. Ann Surg 1996, 224:288-294.

22. Jänne PA, Baldini EH: Patterns of failure following surgical resection for malignant pleural mesothelioma. Thorac Cardiovasc Surg 2004, 1 28: 138-1 46.

23. Rusch VW, Piantadosi S, Holmes EC: The role of extrapleural pneumonectomy in malignant pleural mesothelioma: A Lung Cancer Study Group trial. J Thorac Cardiovasc Surg 1991, 102:I-9.

24. Perrot M, Feld R, Cho BC], Bezjak A, Anraku M, Burkes R, Roberts $H$, Tsao MS, Leighl N, Keshavjee S, Johnston MR: Trimodality therapy with inductin chemotherapy followed by extrapleural pnemonectomy and adjuvant high-dose extrapleural pneumonectomy and adjuvant high-dose hemithoracic radiation for malignant pleural mesothelioma. J Clin Oncol 2009, 27:|4|3-14|8

25. Flores RM, Krug LM, Rosenzweig KE, Venkatraman E, Vincent A, Heelan R, Akhurst T, Rusch VW: Induction chemotherapy, extrapleural pneumonectomy, and postoperative high-dose radiotherapy for locally advanced malignant pleural mesothelioma: A phase III trial. J Thorac Oncol 2006, I:289-295.

26. Rusch VW, Rosenzweig K, Venkatraman E, Leon L, Raben A, Harrison L, Bains MS, Downey RJ, Ginsberg RJ: A phase II trial of surgical resection and adjuvant high-dose hemithoracic radiation for malignant pleural mesothelioma. J Thorac Cardiovasc Surg 200I, 1 22:788-795.

27. Krug LM, Pass HI, Rusch VW, Kindler HL, Sugarbaker DJ, Rosenzweig $\mathrm{KE}$, Flores R, Friedberg JS, Pisters K, Monberg M, Obasaju CK, Vogelzang NJ: Multicenter phase II trial of neoadjuvant pemetrexed plus cisplatin followed by extrapleural pneumonectomy and radiation for malignant pleural mesothelioma. J Clin Oncol 2009 in press.

28. Ball DL, Cruickshank DG: The treatment of malignant mesothelioma of the pleura: Review of I 5-year experience, with special reference to radiotherapy. Am J Clin Oncol 1990, 13:4-9.

29. Linden CJ, Mercke C, Albrechtsson U, Johansson L, Ewers SB: Effect of hemithorax irradiation alone or combined with doxorubicin and cyclophosphamide in $\mathbf{4 7}$ pleural mesotheliomas: $A$ nonrandomized phase II study. Eur Respir J 1996, 9:2565-2572.

30. Mohan R: Dueling technologies: In regard to Ling et al. (Int J Radiat Oncol Biol Phys 2008;72:575-58 I). Int J Radiat Oncol Biol Phys 2009, 75(I):6-7.

31. McCutchen KW, Watkins JM, Eberts P, Terwilliger LE, Ashenafi MS, Jenrette JM 3rd: Helical tomotherapy for total lymphoid irradiation. Radiat Med 2008, 26:622-6.

32. Shi C, Penagaricano J, Papanikolaou N: Comparison of IMRT treatment plans between linac and helical tomotherapy based on integral dose and inhomogenity index. Med Dosim 2008, 33:2I5-21.

33. Sterzing F, Sroka-Perez G, Schubert K, Münter MW, Thieke C, Huber $P$, Debus J, Herfarth KK: Evaluating target coverage and normal tissue sparing in the adjuvant radiotherapy of malignant pleural mesothelioma helical tomotherapy compared with step-and-shoot IMRT. Radiother Oncol 2008, 86:25I-7.

34. Webb WR, Gatsonis C, Zerhouni EA, Heelan RT, Glazer GM, Francis IR, McNeil BJ: CT and MR imaging in staging non-small cell bronchogenic carcinoma report of the Radiologic Diagnostic Oncology Group. Radiology 1991, 178:705-713.

35. Ciernik IF, Dizendorf E, Baumert BG, Reiner B, Burger C, Davis JB, Lütolf UM, Steinert HC, Von Schulthess GK: Radiation treatment planning with an integrated positron emission and computer tomography (PET/CT): a feasibility study. Int J Radiat Oncol Biol Phys 2002, 52:339-50

36. Carlo G, Rosenzweig K, Cascini GL, Tamburrini O: Current status of PET/CT for tumor volume definition in radiotherapy planning for non-small cell lung cancer (NSCLC). Lung Cancer 2007, 57: $125-134$.

37. Bradley J, Thorstad WL, Mutic S, Miller TR, Dehdashti F, Siegel BA, Bosch W, Bertrand RJ: Impact of FDG-PET on radiation therapy volume definition in non-small-cell lung cancer. Int J Radiat Oncol Biol Phys 2004, 59:78-86.

Publish with BioMed Central and every scientist can read your work free of charge

"BioMed Central will be the most significant development for disseminating the results of biomedical research in our lifetime. "

Sir Paul Nurse, Cancer Research UK

Your research papers will be:

- available free of charge to the entire biomedical community

- peer reviewed and published immediately upon acceptance

- cited in PubMed and archived on PubMed Central

- yours - you keep the copyright 